\title{
Modeling on Dry Centrifugal Granulation Process of Molten Blast Furnace Slag
}

\author{
Qingming $\mathrm{CHANG}_{,}{ }^{1) *}$ Xianwang $\mathrm{LI}^{2)}$ Hongwei $\mathrm{NI}^{1)}$ Wenyuan $\mathrm{ZHU}^{2)}$ Chenggang $\mathrm{PAN}^{1)}$ and Shengde $\mathrm{HU}^{1)}$ \\ 1) The Key Laboratory of Refractories and Metallurgy, Wuhan University of Science and Technology, Qingshan District, \\ Wuhan, 430081 P. R. China. $\quad$ 2) China City Environment Protection Engineering Limited Company, Wuchang District,
} Wuhan, 430071 P. R. China.

(Received on February 10, 2015; accepted on February 25, 2015)

\begin{abstract}
Lots of iron molten slag from blast furnace can be produced during iron making process. It is generally cooled by water quenching method which causes many problems, such as water consumption, serious pollution, energy wasting etc. To solve these problems, much more attention has been paid to various dry granulation methods. Among them, dry centrifugal granulation (DCG) method has its own advantages. In this study, a physical and mathematical model of slag flow behavior in DCG process is established. The DCG process with a flat disc at different mass flow rate, temperature or viscosity, surface tension of molten slag, rotating speed and surface roughness of disc is simulated. The influences of various factors on granulation process are analyzed and optimum parameters are obtained. The results show that the granulation can be greatly improved by increasing the rotating speed of the disc and by reducing the molten slag viscosity properly.
\end{abstract}

KEY WORDS: blast furnace slag; dry centrifugal granulation; physical and mathematical model; rotary disc.

\section{Introduction}

Molten slag at high temperature $\left(1300^{\circ} \mathrm{C}-1600^{\circ} \mathrm{C}\right)$ is a byproduct in Steelmaking. To produce one ton of pig iron, about $300 \mathrm{~kg}$ slag is discharged. In traditional process, molten slag is usually quenched rapidly using a large amount of water to obtain glassy-granulated slag. It is then dried before it is used for raw materials of roadbed or cement production. However, this process cause some serious problems which is needed to be overcome, ${ }^{1-4)}$ e.g.: (1) Too much water is consumed to granulate high temperature molten slag. (2) Lots of sensible heat energy contained in high temperature slag is wasted without recovery. (3) Environment would be polluted when molten slag are poured into water pits, not only because alkaline element in slag dissolves in the water, but also because sulfide and dust from slag are emitted into air.

Recently, more and more attention has been paid to dry granulation process of molten slag which can solves the problems mentioned above to a large extent. Different kinds of dry granulation techniques have been proposed and investigated. Typical examples of these processes include granulation by cooling drum, mechanical stirrer, rolling drum, high speed air and centrifugal force etc. ${ }^{1,2)}$ Among them, slag granulation process by a centrifugal force, which is also called DCG process, has proved its advantages due to its simpleness, controllability, less energy consumption and more sensible heat recovery in the past years. ${ }^{5-9)}$ In this

\footnotetext{
* Corresponding author: E-mail: qmchang@163.com

DOI: http://dx.doi.org/10.2355/isijinternational.55.1361
}

process, the key technique is how to obtain small size slag particles for subsequent cement production. For this purpose, the rotary parts have also been studied and designed to different shapes, such as a flat disc, ${ }^{9,10)}$ a deep ${ }^{5-8)}$ or shallow cup etc. ${ }^{10,11)}$ Despite its engineering significance, the phenomena of the slag granulation using DCG process had not yet been theoretically elucidated. Therefore, it is essential to develop a comprehensive model to analyze DCG process, and to investigate the possibility of granulating molten slag by the DCG process with a flat disc. The influence of rotary speed and surface roughness of disc, slag temperature or viscosity, slag surface tension and mass flow rate on the granulation will be is analyzed. Some important suggestions for the possibility of a new slag treatment and for designing the technological parameters of industrial DCG process will be given.

\section{Mathematical Model}

\subsection{Governing Equations of Molten Slag Flow and} Granulation on a Rotary Disc

The DCG process of molten slag is simulated with a three-dimensional simulation program which is based on the VOlume of Fluid (VOF) scheme. ${ }^{12)}$ In this scheme, the fluid flow including molten slag and surrounding gas is described by the momentum and continuity equations, and the transient interface between molten slag and gas is represented by a function which is called volume fraction. The molten slag flow together with surrounding gas is assumed as incompressible two-phase fluid flow, and the Navier-Stokes equations are combined into a single equation in the entire 
domain. Thus, each fluid is taken to be incompressible, and the continuity equation is valid over the whole domain. The governing conservation equations for unsteady, incompressible, liquid-gas flow are given by the following equations:

\subsubsection{Continuous Equation}

$$
\frac{\partial \rho}{\partial t}+\nabla \cdot \vec{V}=0
$$

\subsubsection{Momentum Equation}

$$
\frac{\partial \vec{V}}{\partial t}+(\vec{V} \cdot \nabla) \vec{V}=-\frac{1}{\rho} \nabla p+v \nabla^{2} \cdot \vec{V}+\vec{S}
$$

\subsubsection{Volume of Fluid Equation}

$$
\frac{\partial F}{\partial t}+\nabla \cdot(\vec{V} F)=0
$$

where $\vec{V}$ is the velocity vector, $v$ is the dynamic viscosity, $\rho$ is the density, $\mathrm{p}$ is the pressure, $\vec{S}$ is the momentum source term including gravitational and centrifugal acceleration vector, and $F$ is the volume fraction of fluid in a cell.

Equation (3) expresses that the interface property is advected with the local fluid velocity. In computational cells occupied with liquid phase, $\mathrm{F}$ is unity, and in cells occupied with gas phase, $\mathrm{F}$ is zero. For cells containing the interface bounding liquid and gas phases, $F$ lies between zero and unity. For the local average density and viscosity, linear weighing of the densities of the gas phase and liquid phase are used:

$$
\begin{gathered}
\rho=F \rho_{l}+(1-F) \rho_{g} \\
v=F v_{l}+(1-F) v_{g}
\end{gathered}
$$

where the subscripts 1 and g denote liquid and gas phases, respectively. The expression for density in Eq. (4) results from mass conservation, whereas the expression for mixture viscosity in Eq. (5) is an approximation.

\subsection{Governing Equations of Heat Transfer of Molten Slag and Surround Gas}

Thermal energy balance is given by

$$
\rho c_{p} \frac{\partial T}{\partial t}+\rho c_{p} \vec{V} \cdot \nabla T=\nabla \cdot(k \nabla T)+\rho L \frac{\partial f_{s}}{\partial t}
$$

where $T$ is the temperature, $c_{p}$ is the specific heat, $k$ is the thermal conductivity, $L$ is the latent heat of freezing of the slag, and $f_{s}$ is the fraction of solid.

\subsection{Boundary Conditions}

\subsubsection{Boundary Conditions for Fluid Flow Analysis}

A no slip boundary condition is assumed both at the stationary solid walls and at the rotary disc wall. At the stationary solid walls, the velocity is set to be zero $(\vec{V}=0)$ while at the rotary solid wall, the velocity is set to be the linear speed at the edge of the disc which is the product of the distance $r$ from the location to the center of the disc and the rotary speed $\omega$ :

$$
|\vec{V}|=r \cdot \omega
$$

A parabolic distribution is employed to assign inlet flow velocity of the slag, which is equivalent to the expected mass flow rate of molten slag. The outlet boundary of the calculation zone is assumed to be a constant pressure outlet which is referred to the pressure in the DCG vessel.

\subsubsection{Boundary Conditions for Heat Transfer Analysis}

A zero heat flux boundary condition is set on the inlet and outlet boundaries, and a Cauchy boundary condition is adopted at molten slag-gas interfaces:

$$
q=h\left(T-T_{r e f}\right)+\sigma \varepsilon\left(T^{4}-T_{r e f}^{4}\right)
$$

where $h$ indicates the heat transfer coefficient between molten slag and surrounding gas, $T$ describes the temperature of the slag-gas interface, and $T_{r e f}$ describes the temperature of the gas. $\sigma$ indicates the Boltzmann constant and $\varepsilon$ indicates the emissivity and is set to be 0.8 .

\section{Experimental Setup}

Figure 1 shows the experimental apparatus of DCG process for molten slag and Fig. 2 indicates the corresponding schematic diagram. The disc driven by a motor at the base is placed in a big vessel with cooling water. The molten slag in a graphite crucible is heated in an electric resistance stove. This crucible with a plug closing the bottom hole is positioned above the rotary disc. Air pressure is employed on the plug to hold it in position until the molten slag is poured. A pneumatic cylinder is activated by remote control and raises the graphite rod or plug. This allows the molten slag to flow through a $10 \mathrm{~mm}$ diameter opening, onto the disc with an expected mass flow rate. The slag falls under the gravity force before impinging the disc and then spreads out to granulate. This procedure is recorded by a high-speed video camera shown in Fig. 2.

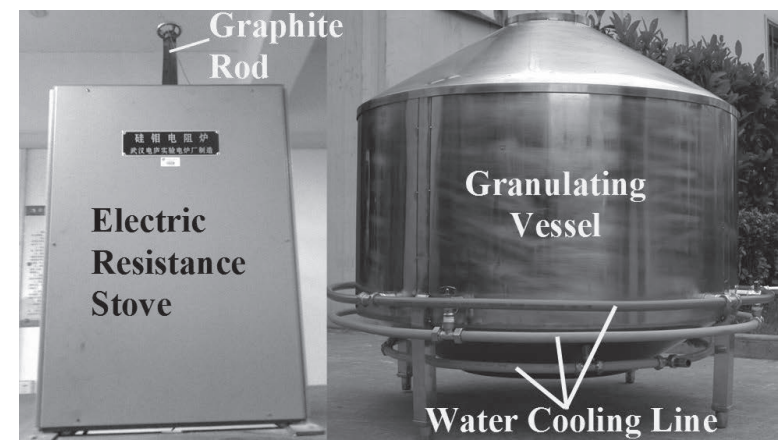

Fig. 1. Experimental apparatus of DCG process for molten slag.

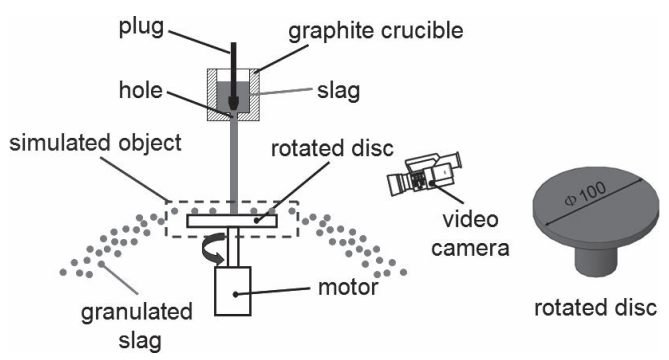

Fig. 2. Schematic diagram of experimental apparatus. 


\section{Results and Discussions}

\subsection{Model Verification}

To verify the model before further investigation, the calculation is demonstrated to predict the particle diameter of the granulated slag particles and the modeling results are compared with experimental results. In the experiment, the slag is remelted and maintained at temperature about $1500^{\circ} \mathrm{C}$. The disc with $200 \mathrm{~mm}$ diameter spins at a rotating speed of $1500 \mathrm{r} / \mathrm{min}$. The rod is unplugged and molten slag is poured from the crucible. The experiment is observed from video camera records and the granulated slag particles are collected on a peculiar collecting sheet. Figure 3 exhibits the experimental photographs of dry centrifugal granulation process and the collected slag particles. In Fig. 3(b), small particles with yellow color are the droplets which directly solidify after they leave the edge of the disc. The glass ratio of these slag particles is higher than $98 \%$. The big particles with dark color are those which bond together after they leave the disc and hit the vessel wall. The glass ratio of these particles is higher than $92 \%$, which satisfy the requirement for producing cement.

Because the granulated slag drop is very small (few millimeter), and the granulation vessel is very large (for our experimental setup, its diameter is about 3 meters), only a small region near the disc shown in Fig. 3 is taken for simulating the granulation process in order to shorten the calculation time. At this small region, the modeling is carried out under the same conditions. Many calculations have been tried and exhibit that the slag temperature near the disc changes so small that the granulation process can be considered as a quasi isothermal process. For example, as shown in Fig. 4, the lowest temperature at the surface of the

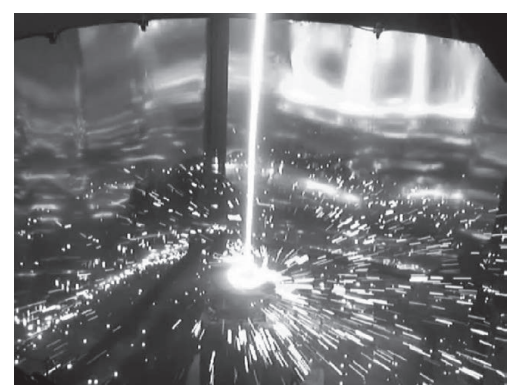

(a)

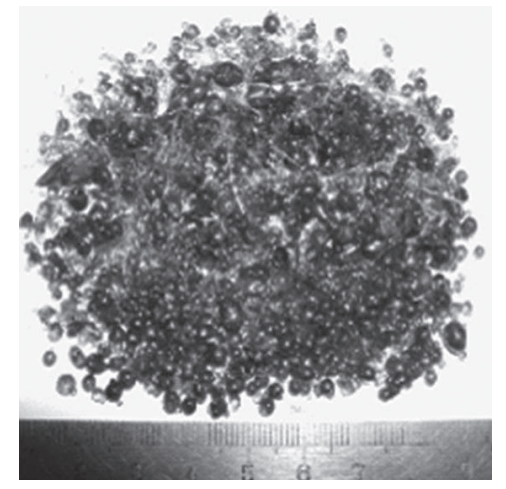

(b)

Fig. 3. Experiment of dry centrifugal granulation process. (a) granulation process of molten slag. (b) granulated particles. droplet is about $1390^{\circ} \mathrm{C}$, which is about $110^{\circ} \mathrm{C}$ drop from its initial temperature $\left(1500^{\circ} \mathrm{C}\right)$ and higher than the liquidus temperature of slag $\left(1380^{\circ} \mathrm{C}\right)$. That is to say, during the total granulation process of simulation, the slag remains liquid. Thus, the physical properties of the slag are simplified as constants and their values at $1500^{\circ} \mathrm{C}$ are adopted at all simulations in this paper. The heat transfer and temperature distribution during this granulation period are really insignificant. Therefore, the subsequent part of this paper focuses only on the granulation process without heat transfer in order to reduce the calculation time consumption.

Table 1 gives some properties of slag at $1500^{\circ} \mathrm{C}$. As shown in Fig. 4 in which the modeling approaches a quasi steady state, the molten slag starts out as a column flowing from the slag inlet (bottom holes of the crucible) and falls on the center of the rotary disc. Then it spreads out on the disc due to centrifugal force to form a thin film and a number of ligaments soon. Thereafter, these ligaments broke into many small droplets and became granulates under the effect of surface tension, gravity force and centrifugal force. It is not difficult to imagine that the size of the droplet, the thin round film and length of the ligaments is influenced by lots of factors such as the rotary speed, viscosity, pouring flow rate etc., which will be investigated later in this paper.

The comparison of diameter distribution of granulated slag particles between numerical and experimental results is given in Fig. 5. Both in simulation and in experiment,

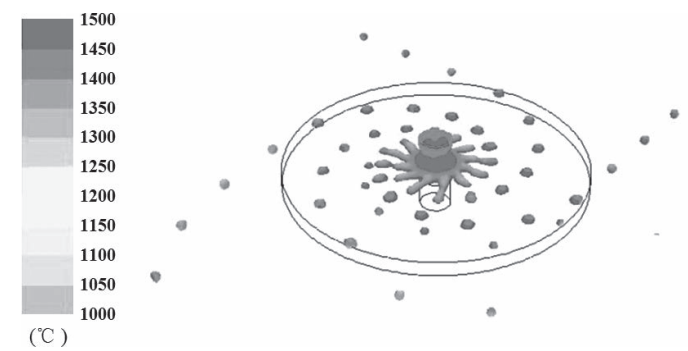

Fig. 4. Modeling of DCG process.

Table 1. Thermophysical properties of molten slag at $1500^{\circ} \mathrm{C}$.

\begin{tabular}{ccccc}
\hline Materials & $\begin{array}{c}\text { Density } \\
\left(\mathrm{kg} / \mathrm{m}^{3}\right)\end{array}$ & $\begin{array}{c}\text { Viscosity } \\
(\mathrm{Pa} \mathrm{s})\end{array}$ & $\begin{array}{c}\text { Specific heat } \\
(\mathrm{kJ} / \mathrm{kg} / \mathrm{K})\end{array}$ & $\begin{array}{c}\text { Thermal } \\
\text { conductivity } \\
(\mathrm{W} / \mathrm{m} / \mathrm{K})\end{array}$ \\
\hline slag & 2900 & 0.45 & 1.2 & 0.1 \\
\hline
\end{tabular}

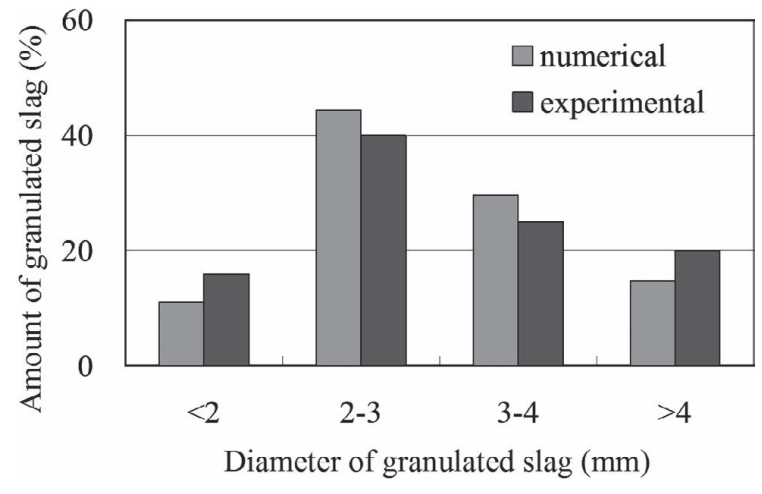

Fig. 5. Comparison of diameter distribution of granulated slag particles between numerical and experimental results. 


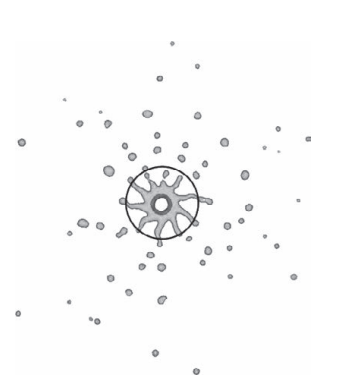

(a)

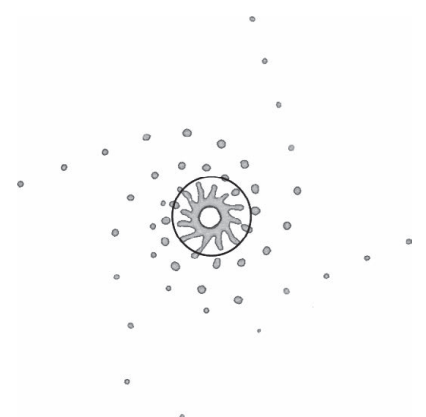

(b)

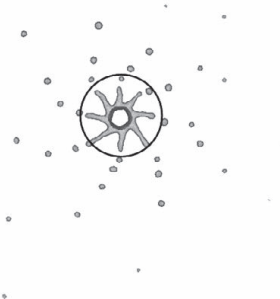

(c)

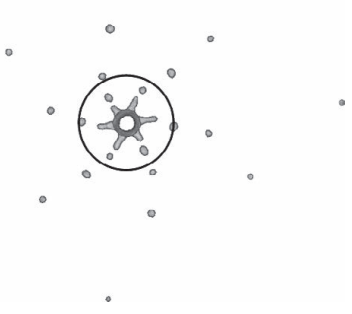

(d)

Fig. 6. Effect of rotary speed on DCG process. (a) $500 \mathrm{r} / \mathrm{min}$. (b) $1500 \mathrm{r} / \mathrm{min}$. (c) $3000 \mathrm{r} / \mathrm{min}$. (d) $5000 \mathrm{r} / \mathrm{min}$.

more than $65 \%$ in wt of the slag particles ranges from 2 $\mathrm{mm}$ to $4 \mathrm{~mm}$ in diameter, which can meet the requirements for producing cement or roadbed materials. Furthermore, it is obvious that the simulated results agree well with the experimental ones.

\subsection{Effect of Rotary Speed of the Disc on Granulation Process}

As mentioned above, there are lots of factors which influence the granulation process. One important of them is the rotating speed of the disc. The granulation processes at different rotary speed are shown in Fig. 6. In each case, a $200 \mathrm{~mm}$ in diameter disc at different rotary speed is modeled with a pouring temperature $1500^{\circ} \mathrm{C}$ and mass flow rate $0.35 \mathrm{~kg} / \mathrm{s}$. It is easily observed that, both the size of the round thin film and the particle diameter are reduced with a faster rotary speed. However, the number of the ligaments increases with the rotary speed at its lower value (e.g. less than $2000 \mathrm{rpm}$ ), and decreases at its higher value. It is not difficult to understand this phenomenon. At a lower rotary speed, the thickness of the film takes the main effect on the number of the ligaments. At this condition, a faster rotary speed can reduced the thickness of the film and thus increase the number of the ligaments under the same pouring flow rate; On the hand, at a higher rotary speed, the radial velocity of the of the molten slog in the ligaments produces more effects on the number of the ligaments. At this time, a faster rotary speed can accelerate the radial velocity of the slag and thus reduce the number of the ligaments under the same pouring flow rate. This indicates that the centrifugal force plays an important role in granulation process of molten slag. Figure 7 gives the corresponding relationship between the mean diameter of granulated slag and disc rotating speed. When the rotating speed is slower, e.g., less than $2000 \mathrm{rpm}$, the diameter of the granulated slag particles decreases rapidly with the increased rotating speed. However, when the rotating speed is faster than $2000 \mathrm{rpm}$, the diameter of the granulated slag particles varies slightly with the rotating speed, and finally approaches to a constant, indicating that to obtain fine slag particles, $2000 \mathrm{rpm}$ is optimum rotating speed for granulation process in this case.

The diameter distribution of granulated slag particles at different rotating speed is shown in Fig. 8. It is observed that more fine slag particles can be obtained with a faster rotating speed.

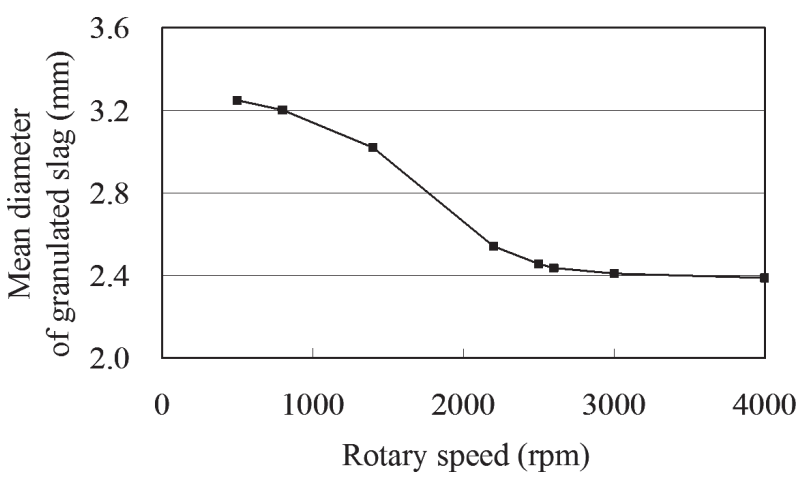

Fig. 7. Effect of rotating speed on DCG process.

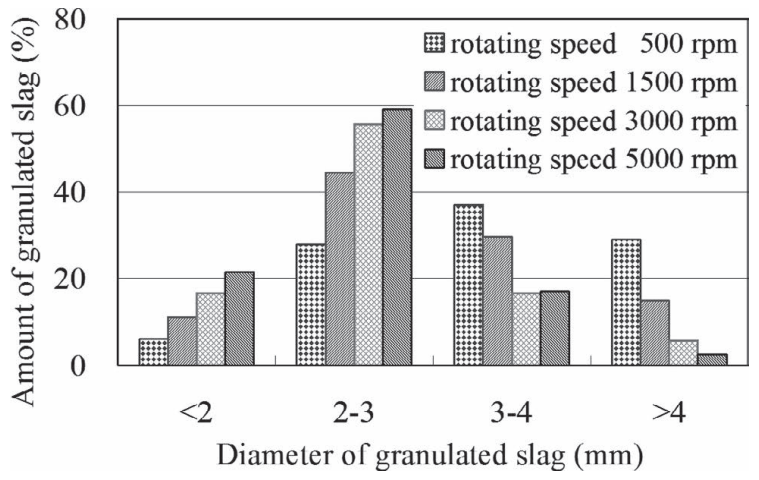

Fig. 8. Diameter distribution of granulated slag particles at different rotating speed.

\subsection{Effect of Pouring Temperature (or Viscosity) on Granulation Process}

Pouring temperature is another important factor which influences the granulation process of molten slag. Generally, lots of physical properties of molten slag vary with molten temperature. These physical properties include viscosity, density, specific heat and etc. However, compared with the influence of temperature on molten slag viscosity, the influence of temperature on other physical properties is so small that it can be ignored. The variation of viscosity with temperature is measured by a rotating viscosimeter and shown in Fig. 9. The molten slag viscosity varies slowly with temperature at high temperature while it increases sharply with temperature variation when the temperature is below $1400^{\circ} \mathrm{C}$. For a given composition slag, its viscosity is suggested to be a unique function of temperature. From 


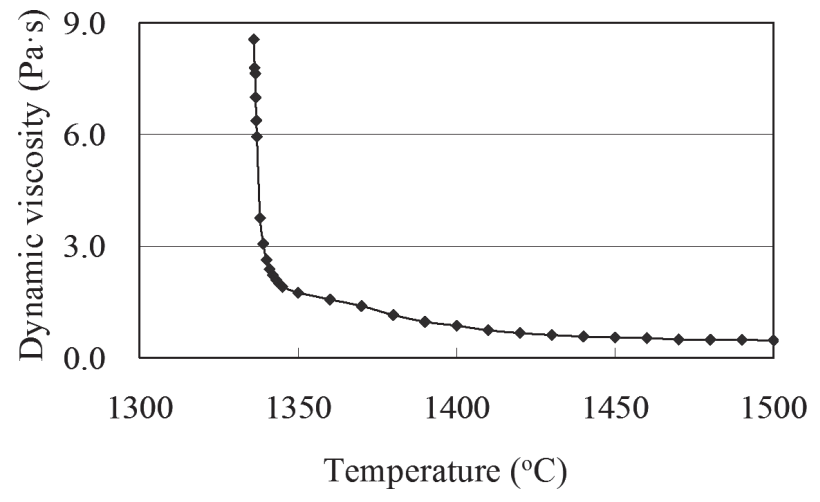

Fig. 9. Viscosity variation with melt temperature.

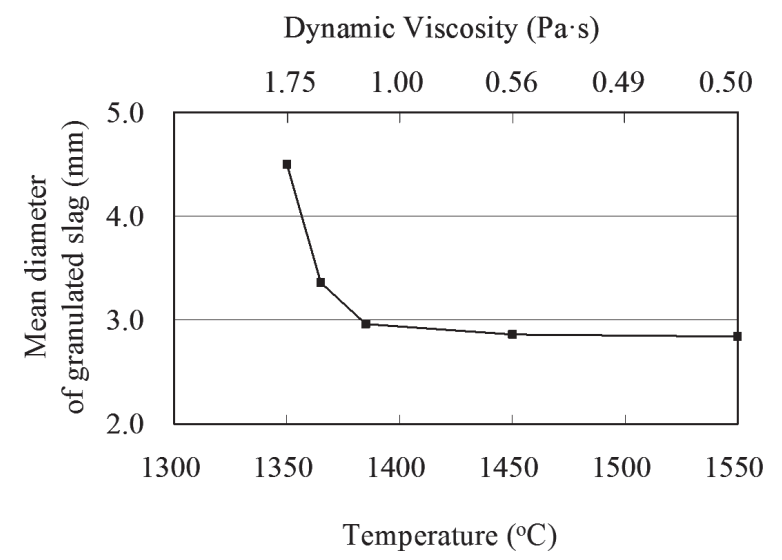

Fig. 10. Effect of molten slag temperature and viscosity on mean diameter of granulated slag.

this point, the effect of pouring temperature on granulation process is equivalent to that of molten slag viscosity.

Both disc rotating speed and mass flow rate are kept constant at $1500 \mathrm{rpm}$ and $0.35 \mathrm{~kg} / \mathrm{s}$ respectively, and the disc diameter is set to be $200 \mathrm{~mm}$. This allows an assessment of the effect of the pouring temperature (or viscosity) on granulation process. Figure 10 displays the mean diameter variation of the granulated slag particles with molten slag viscosity and temperature. As expected, with increasing temperature or decreasing viscosity the mean diameter of the slag particles decreases rapidly at relatively lower temperature $\left(1400^{\circ} \mathrm{C}\right)$ or at relatively higher viscosity, and varies slowly at relatively higher temperature or at relatively lower viscosity. Therefore, to obtain fine granulated slag particles, the pouring temperature or viscosity should be controlled.

\subsection{Effect of Disc Diameter on Granulation Process}

The effect of disc diameter on granulation process is also studied with pouring temperature $1500^{\circ} \mathrm{C}$, pouring mass flow rate $0.8 \mathrm{~kg} / \mathrm{s}$ and disc rotating speed $1500 \mathrm{rpm}$. The result is plotted in Fig. 11. It is not difficult to find that the disc diameter has obvious effect on granulation process. With a bigger diameter, a higher linear speed at the outer edge of the disc can be reached, and thus the molten slag is more easily broken, and finer particles can be produced. However, more power and energy are required to drive a bigger disc, and therefore the disc size should be limited to an appropriate value.

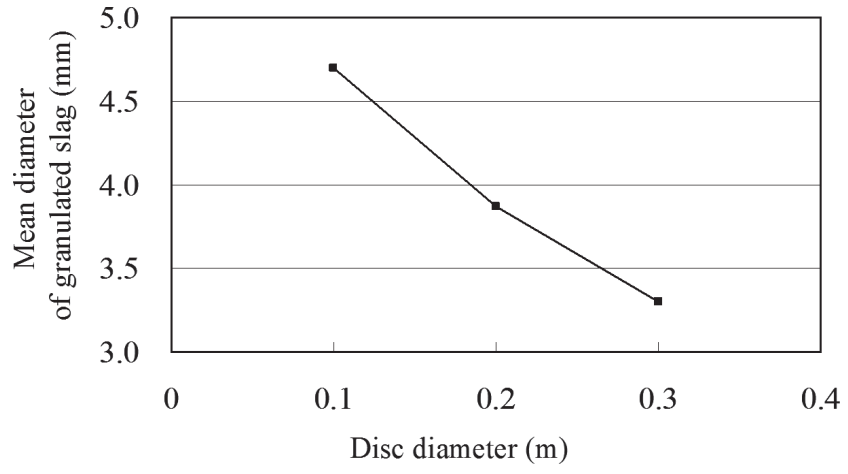

Fig. 11. Effect of rotary disc diameter on mean diameter of granulated slag.

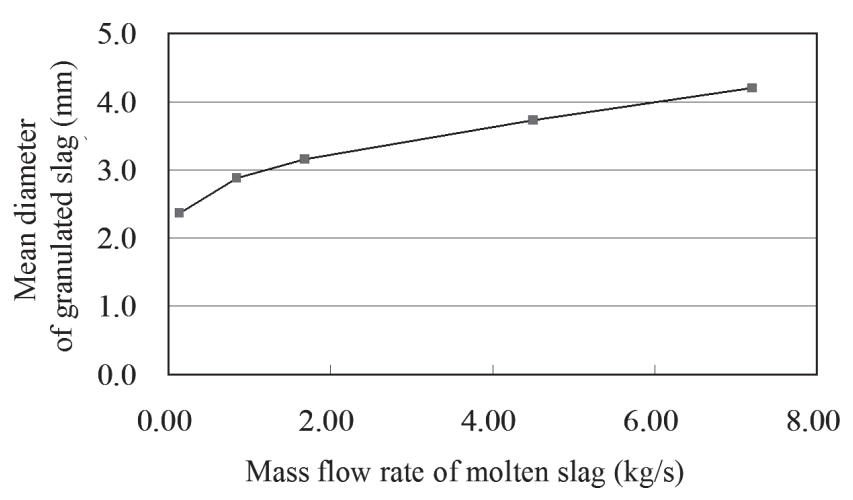

Fig. 12. Effect of slag mass flow rate on mean diameter of granulated slag.

\subsection{Effect of Molten Slag Mass Flow Rate on Granula- tion Process}

The effect of molten slag mass flow rate on the mean diameter of granulated slag particles is simulated with pouring temperature $1500^{\circ} \mathrm{C}$, disc rotating speed $1500 \mathrm{rpm}$ and disc diameter $200 \mathrm{~mm}$ and plotted in Fig. 12. The diameter became larger with increasing molten slag mass flow rate. This tendency becomes more obvious at relatively small molten slag mass flow rate. It is easy to conclude that the molten slag mass flow rate must be controlled in order to obtain expected fine slag particles for industrial granulation process.

\subsection{Effect of Surface Tension of Molten Slag on Gran- ulation Process}

Surface tension of the molten slag is not only related to temperature, but also to the composition of the slag. How the composition of the slag influences the granulation process through the surface tension is discussed in the next part.

Keeping the other conditions fixed except the surface tension of the molten slag, the mean diameter of granulated slag particles can be calculated under different surface tensions of the molten slag, and their relationship is given in Fig. 13. Clearly, the mean diameter significantly decreases with a lower surface tension applied and slightly decreases when the surface tension is less than $0.45 \mathrm{~N} / \mathrm{m}$. It is not difficult to understand that a lower surface tension indicates a smaller inner cohesive force which can help the molten slag to break into fine droplets. 


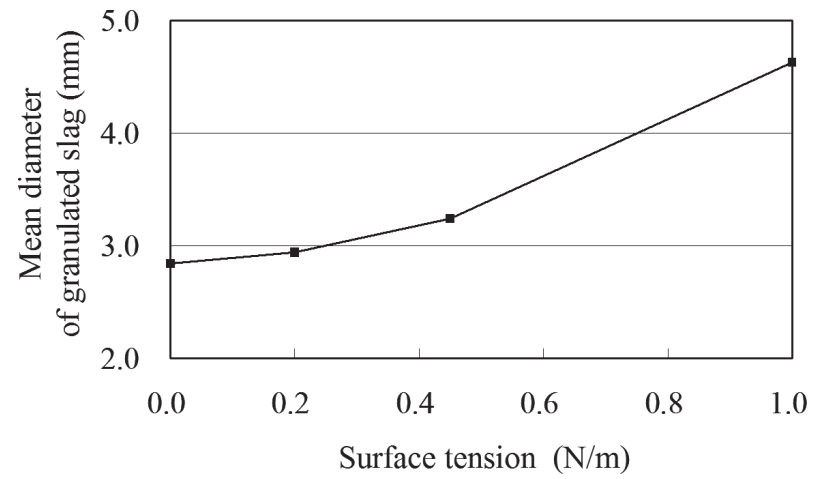

Fig. 13. Effect of surface tension of molten slag on mean diameter of granulated slag.

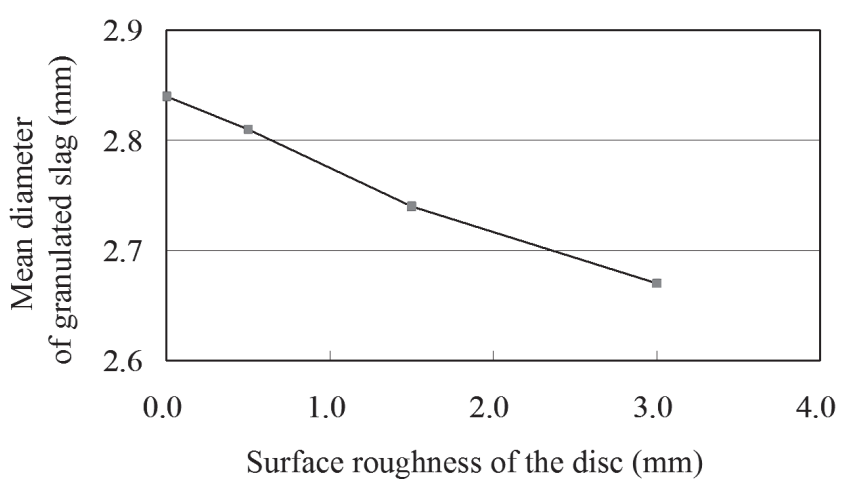

Fig. 14. Effect of disc surface roughness on mean diameter of granulated slag particles.

\subsection{Effect of Roughness of Rotary Disc on Granulation Process}

The surface roughness of the rotary disc presents the friction between the disc surface and molten slag, and thus influences the granulation process. In this paper, the surface roughness is defined as the contour arithmetic mean deviation $\mathrm{Ra}$. In the calculations, the molten slag at $1500^{\circ} \mathrm{C}$ is poured on a rotating disc with a $200 \mathrm{~mm}$ diameter at $1500 \mathrm{rpm}$ speed. The mass flow rate is controlled at 0.35 $\mathrm{kg} / \mathrm{s}$. Figure 14 gives the relationship between the surface roughness of the rotary disc and the mean diameter of the granulated slag particles. It can be observed that the mean diameter is inversely proportional to the surface roughness of the disc. That is to say, the larger the surface roughness of the disc, the bigger the friction force exerted on the molten slag and thus the finer the granulated slag particles. However, compared with that of other factors, the effect of disc roughness on the mean diameter is so small that it can be ignored for DCG process. For example, if the surface roughness of the disc is set to be $0 \mathrm{~mm}, i$. e., the disc owns an ideal smooth surface, slag particles with a $2.84 \mathrm{~mm}$ mean diameter can be obtained. If the roughness is increased to $3.0 \mathrm{~mm}$, the mean diameter of the slag particles is reduced to $2.67 \mathrm{~mm}$, i.e. the mean diameter is only $6 \%$ reduced. Moreover, in the experiment, filament phenomenon of molten slag is readily observed during granulation process with

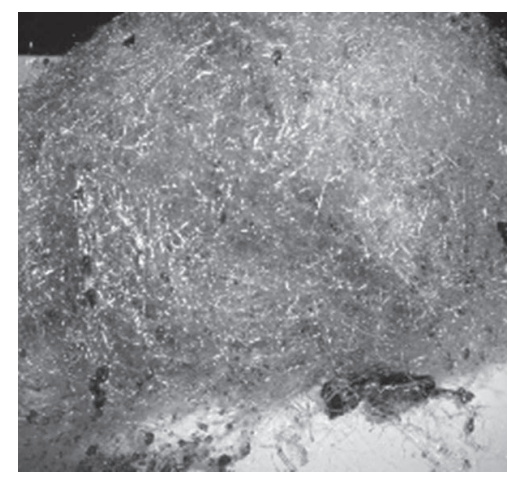

Fig. 15. Filament fibers produced with a rough surface disc during DCG process.

a rough surface disc, as seen in Fig. 15, which is undesired in industrial application. So, a relatively smooth surface for industrial granulation process is a better choice.

\section{Conclusions}

(1) A model for flow and granulation process of molten slag on the rotary disc is established and validated by experiment. Six factors including rotary speed of disc, diameter of the disc, molten slag temperature or viscosity, slag mass flow rate, surface tension of molten slag and surface roughness of disc are analyzed with this model. The results show that the former four factors are more important relatively compared with the other two factors.

(2) To obtain fine slag particles, a higher rotary speed of disc, higher temperature or lower viscosity, bigger diameter of disc and lower surface tension of molten slag should be applied for industrial granulation process, and discs with a smooth surface should be selected to avoid filamentary slag, even it may lead to coarsening of granulated slag particles.

\section{Acknowledgements}

The authors would like to acknowledge the National Natural Science Foundation of China (51074116) and key project funded by the Education Department of Hubei Province (D20101110).

\section{REFERENCES}

1) G. Bisio: Energy, 22 (1997), 501.

2) M. Barati, S. Esfahani and T. A. Utigard: Energy, 36 (2011), 5440

3) S. J. Picketing, N. Hay, T. F. Roylanae and G. H. Thomas: Ironmaking Steelmaking, 12 (1985), 14.

4) H. T. Shen and E. Forssberg: Waste Manage., 23 (2003), 933.

5) N. Maruoka, T. Mizuochi, H. Purwanto and T. Akiyama: ISIJ Int., 44 (2004), 257.

6) T. Mizuochi, T. Akiyama, T. Shimada, E. Kasai and J. Yagi: ISIJ Int., 41 (2001), 1423

7) H. Purwanto, T. Mizuochi, H. Tobo, M. Takagi and T. Akiyama: Mater. Trans., 45 (2004), 3286.

8) H. Purwanto and T. Akiyama: Energy, 31 (2006), 491.

9) H. Purwanto, T. Mizuoch and T. Akiyama: Mater. Trans., 46 (2005), 1324.

10) W. Xie, Y. Y. Zhao and J. J. Dunkley: Powder Metall., 47 (2004), 168.

11) T. Mizuochi and T. Akiyama: ISIJ Int., 43 (2003), 1469.

12) C. W. Hirt and B. D Nichols: J. Comput. Phys., 39 (1981), 201. 